\title{
BMJ Open Reducing social isolation and loneliness in older people: a systematic review protocol
}

Filipa Landeiro, Paige Barrows, Ellen Nuttall Musson, Alastair M Gray, José Leal

To cite: Landeiro F, Barrows $P$, Nuttall Musson E, et al. Reducing social isolation and loneliness in older people: a systematic review protocol. BMJ Open 2017;7:e013778. doi:10.1136/ bmjopen-2016-013778

- Prepublication history and additional material are available. To view these files please visit the journal online (http://dx.doi. org/10.1136/bmjopen-2016013778).

Received 5 August 2016 Revised 24 0ctober 2016 Accepted 25 November 2016

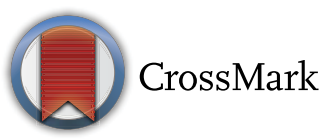

Health Economics Research Centre, Nuffield Department of Population Health, University of Oxford, Oxford, UK

Correspondence to

Miss Filipa Landeiro;

filipa.landeiro@dph.ox.ac.uk

\section{ABSTRACT}

Introduction Social isolation and loneliness affect approximately one-third to one-half of the elderly population and have a negative impact on their physical and mental health. Group-based interventions where facilitators are well trained and where the elderly are actively engaged in their development seem to be more effective, but conclusions have been limited by weak study designs. We aim to conduct a systematic review to assess the effectiveness of health promotion interventions on social isolation or loneliness in older people.

Methods and analysis A systematic review was conducted in Medline, Embase, PsycINFO, Cumulative Index to Nursing and Allied Health Literature, Applied Social Sciences Index and Abstracts, LILACS, OpenGrey and the Cochrane Library on peer-reviewed studies and doctoral theses published between 1995 and 2016 evaluating the impact of health promotion interventions on social isolation and/or loneliness for individuals aged 60 and over. Two reviewers will independently assess each study for inclusion and disagreements will be resolved by a third reviewer. Data will be extracted using a predefined pro forma following best practice. Study quality will be assessed with the Effective Public Health Practice Project quality assessment tool. A narrative synthesis of all studies will be presented by type of outcome (social isolation or loneliness) and type of intervention. If feasible, the effectiveness data will be synthesised using appropriate statistical techniques.

Ethics and dissemination This systematic review is exempt from ethics approval because the work is carried out on published documents. The findings of the review will be disseminated in a related peer-reviewed journal and presented at conferences. They will also contribute to a DPhil thesis.

Trial registration number CRD42016039650

\section{INTRODUCTION}

Worldwide, the population is ageing as a result of decreasing mortality and fertility. ${ }^{1}$ The global share of individuals aged 60 years or over represented $11.7 \%$ of the world population in 2013 and is expected to reach $21.1 \%$ by $2050 .{ }^{2}$ Up to $50 \%$ of those aged over 60 are at risk of social isolation ${ }^{3}$ and approximately one-third of older people will experience some degree of loneliness later in life. ${ }^{45}$
Strengths and limitations of this study

- This systematic review of interventions to alleviate social isolation or loneliness was based on a detailed search strategy including studies from any country published in any language.

- The review followed robust guidelines and the quality of the papers included was assessed using a validated tool.

- The heterogeneity of the interventions and of the tools used to measure social isolation or loneliness may not allow for direct comparisons between studies.

Social isolation has detrimental effects on health,${ }^{67}$ having been identified as a risk factor for all-cause morbidity and mortality ${ }^{8}$ with outcomes comparable to smoking, obesity, lack of exercise and high blood pressure. ${ }^{9}$ It has also been associated with decreased resistance to infection, ${ }^{10}{ }^{11}$ cognitive decline and mental health conditions such as depression and dementia ${ }^{9}$ and with increased emergency admission to hospital, ${ }^{12}$ longer length of stay and delayed discharges. ${ }^{13}$

The literature identifies two main types of interventions aiming to reduce social isolation and loneliness: group-based interventions (ie, support groups, ${ }^{1415}$ reminiscence therapy, ${ }^{1617}$ videoconferencing, ${ }^{18}$ ) and one-to-one interventions (ie, computer use training, ${ }^{19}$ animal companionship, ${ }^{20}{ }^{21}$ visitor volunteers, ${ }^{22}$ etc). These types of interventions can be implemented in the community (ie, in a centralised location, such as centres for adult education or at the participant's home) or in a supported living facility (ie, nursing or residential homes, warden-controlled flats, etc). These interventions can focus on: social skills training (ie, educational course on friendship, ${ }^{23}$ strategies to develop social behaviours, ${ }^{24}$ etc); enhanced social support (ie, befriending volunteer programme, ${ }^{22}$ support groups following bereavement, ${ }^{15}$ etc); increased opportunity for social interaction 
(ie, through the provision of services such as transport, ${ }^{25}$ home-delivered meals ${ }^{26}$ and use of technology such as internet and interactive games or activities, ${ }^{27-30}$ etc); and social cognitive training (ie, self-management group sessions, ${ }^{31}$ etc). Furthermore, these interventions can either be technology assisted or not.

Previous reviews of health promotion interventions aimed at reducing social isolation in the elderly suggest that interventions with group-based formats and where individuals are required to actively participate were more effective than one-to-one interventions. ${ }^{3233}$ Also, involving the study participants in the planning, implementation and evaluation of policies, ${ }^{34}$ high-quality training of facilitators $^{32}$ and interventions based on existing community resources seem to produce more successful outcomes. ${ }^{44}$ The individuality of the experience of loneliness is an important issue which has also been highlighted in the literature, as this may cause difficulty in the delivery of standardised interventions: it has been suggested that programmes which are tailored to meet individual needs may be more appropriate and successful. ${ }^{4}$ But previous reviews were restricted to studies published in English language ${ }^{33-38}$ and up to 2013. Furthermore, statistical synthesis of effectiveness data has been largely lacking, ${ }^{33} 34363839$ as well as the assessment of the quality of the studies included using a validated tool. ${ }^{34-36}{ }^{39}$ Previous systematic reviews that assessed the quality of the studies suggest that the literature investigating the effectiveness of interventions aiming to reduce social isolation or loneliness is of poor methodological quality and, although conclusions have been drawn, further investigation is required. ${ }^{32}$ The aim of this review is therefore to identify health promotion interventions aiming to alleviate social isolation or loneliness in older people and to assess their effectiveness.

\section{METHODS AND ANALYSIS}

\section{Protocol and registration}

We followed the reporting guidelines of the Preferred Reporting Items for Systematic Reviews and Meta-Analysis for Protocols 2015 (PRISMA-P). ${ }^{40}$ The completed PRISMA-P checklist is provided in online supplementary file 2. The protocol is registered with the PROSPERO international prospective register of systematic reviews (registration number CRD42016039650). The final review will be reported following the PRISMA statement. ${ }^{41-43}$ Important amendments to this protocol will be reported and published with the results of the review.

\section{Study selection criteria}

Type of participants

Studies will be included if the full or part of the study population is older persons. The agreed United Nations cut-off age of 60 years will define the older population. ${ }^{1}$

\section{Type of studies}

This systematic review will include studies published in a peer-reviewed journal or doctoral thesis using a randomised control trial (RCT), non-randomised controlled trial (NRCT), controlled before-and-after (CBA) or uncontrolled before-and-after (BA) study design.

\section{Type of outcome measure}

The outcome of interest is social isolation or loneliness measured using appropriate instruments. Both validated (eg, Lubben Social Network Scale ${ }^{44}$ and Duke Social Support Index ${ }^{4546}$ to measure social isolation and De Jong Gierveld Scale ${ }^{47}$ and UCLA Loneliness Scale ${ }^{48}$ to measure loneliness) and non-validated outcome instruments of social isolation or loneliness will be considered. To be included, studies must report a quantitative measure of the effect of the health promotion intervention on social isolation or loneliness.

\section{Type of intervention}

Studies will only be included if the health promotion intervention under analysis was designed specifically to alleviate or prevent social isolation or loneliness.

\section{Search strategy}

\section{Electronic databases}

The selection of electronic databases and the search strategy were developed in conjunction with an information specialist and were based on previous literature reviews' search strategies. ${ }^{33} 3949$ The following electronic databases were searched from 1995 until the end of 2015: Medline, Embase, PsycINFO, Cumulative Index to Nursing and Allied Health Literature, Applied Social Sciences Index and Abstracts, LILACS, OpenGrey and the Cochrane Library. No language or geography restrictions were applied to the search. The exact search terms used in all databases are described in online supplementary file 1 .

\section{Manual searches}

The reference list of the studies included in this review, as well as those of previous literature reviews on health promotion interventions to reduce social isolation or loneliness, will be searched to identify additional potentially relevant studies.

\section{Study selection}

ENDNOTE X7, Thomson Reuters, will be used to manage the references. Duplicates will be removed by one reviewer (FL). Two reviewers (FL and PB) will then independently assess each abstract to determine whether full-text review is needed. Any disagreement between the two reviewers will be resolved by a third reviewer (JL). Full text of potentially eligible studies will be retrieved and reviewed and assessed for final inclusion by two reviewers (FL and $\mathrm{PB}$ ) again with a third reviewer (JL) being consulted if necessary. Following PRISMA guidelines, ${ }^{41}$ a flow diagram will be created to illustrate the selection process.

\section{Data extraction}

Data extraction will be conducted independently by two authors (FL and $\mathrm{PB}$ ) and disagreements will be solved as 
described above. Non-English references will be reviewed by two native or fluent speakers. The following information will be extracted using a data extraction form based on 'The Cochrane Group Data collection form for intervention reviews' ${ }^{50}$ Data extracted will cover the following points (see online supplementary file 3 ):

- Study details: title, author, publication details, location, language (if not English);

- Study design: type of study, duration, outcomes measured;

- Participant demographics: setting, inclusion and exclusion criteria, population size and demographics;

- Intervention characteristics: duration, type and mode of intervention;

- Outcomes: measure of outcome used, any other outcomes analysed;

- Results: raw data and effect size for social isolation or loneliness as main outcome as well as secondary outcomes;

- Conclusions: author and reviewer conclusions.

\section{Risk of bias (quality) assessment}

Two reviewers (FL and PB) will perform a quality appraisal of each study independently using the Effective Public Health Practice Project 'Quality assessment tool for quantitative studies" ${ }^{51}$ recommended by the Cochrane Public Health Group as it is applicable to both experimental and quasiexperimental study designs. ${ }^{52}$ Non-English references will be reviewed by two native or fluent speakers for the quality assessment.

\section{Description of studies and measurements of effect size}

We expect to find a diverse range of study designs and heterogeneous interventions aimed at social isolation or loneliness. Hence, data will be divided by type of outcome: impact of interventions on social isolation and impact of interventions on loneliness. Social isolation and loneliness are intricately related but distinct concepts which are frequently used interchangeably. ${ }^{4}$ Social isolation is defined as a scarcity of contacts or social encounters of adequate quality or quantity, and is regarded as an objective measure of social interaction, ${ }^{63953} 54$ whereas loneliness is described as the subjective counterpart of social isolation, where an individual's perceived level of interaction with others does not fulfil their expectations, often resulting in an unpleasant emotional experience. ${ }^{45556}$ Given that these two concepts have been used interchangeably and inconsistently in the literature, we will consider both collectively for search purposes but separately in terms of analysis. Similarities and differences found in the literature will be compared and discussed.

We will further divide the studies by type of study design (eg, RCT, NRCT, CBA and BA) and subdivide them by type of intervention (eg, group, one to one and other designs). A narrative synthesis of all relevant studies will be provided by type outcome, divided in terms of study design and subdivided by type of intervention, describing study and participants' characteristics, interventions, outcomes, results and author's conclusions.

The effectiveness of the health promotion interventions on alleviation or prevention of social isolation or loneliness will be presented in terms of mean effect size (eg, standardised mean difference) and respective CI. The rationale for these summary statistics is the expected variation in the instruments used to assess the same outcome. The effect size will be calculated using Hedges' (adjusted) $\mathrm{g}$, as it provides a superior estimate of the standardised mean difference (SMD) in studies with small samples. ${ }^{57}$

The primary effect size for each study will be calculated from the first available postintervention measurement time point. If a study has more than one intervention, the primary effect size will be calculated for the main intervention group targeting social isolation or loneliness or the group with the most robust design (eg, the intervention which yields the largest difference from the control group).$^{35}$ If a study has more than one control group, the primary effect size will be calculated using the group which theoretically is expected to generate the greatest difference from the intervention group. ${ }^{35}$ In cases where there are more than two groups, we will first conduct pairwise comparisons and also explore more complex analysis, if appropriate, as suggested by Cochrane. ${ }^{58}$

The authors of the studies included in this review will be contacted with the aim to retrieve any missing data necessary for our analysis. We will attempt to calculate any missing SMDs for continuous measures from the reported statistics (eg, CI and SEs) in the relevant paper.

If sufficient data are available, subgroup analysis (ie, type of intervention) will be conducted to account for heterogeneity. If there are sufficient numbers of comparisons for the same outcome and intervention across studies, the between-study heterogeneity will be quantified by calculating the $\chi^{2}$ test for heterogeneity (significance level $\mathrm{p}<0.1$ ) and the $\mathrm{I}^{2}$ statistic. We will report the sum of the studies using both a fixed-effect and random-effects meta-analysis by type of study design and intervention.

\section{DISCUSSION}

This systematic review will be performed to compare the effectiveness of health promotion interventions in alleviating social isolation or loneliness in older persons. By grouping interventions, we will be able to determine which type of intervention is more likely to be effective and we will also assess the role technology plays in promoting social contacts. We will use a validated tool to assess the quality of evidence since previous reviews refer they were limited by the weak methodology of studies analysed, and we will synthesise the data using appropriate statistical methods, if feasible. Furthermore, we will include studies conducted in the last 20 years without any languages or any geographic restrictions. Previous reviews were restricted to studies published in English language and up to 2013. 
Our review aims to address an increasingly relevant problem in terms of the impact it has on older people's health and on health and social care systems worldwide. This review will therefore provide policy makers with a better insight on how to tackle social isolation and loneliness by identifying the type of interventions that alleviate or prevent social isolation or loneliness and under which circumstances.

Acknowledgements The publication of this article was funded by the 0pen Access fund of the Nuffield Department of Population Health, University of Oxford. We would also like to thank our information specialists Eli Harriss and Nia Roberts for their help in developing the search strategy and selecting databases.

Contributors FL conceived the initial idea for the study and is the guarantor of the review. FL and PB wrote the protocol. ENM, AMG and JL critically appraised the protocol and also contributed to its development by revising different versions. All authors read and approved the final version of the manuscript.

Competing interests None declared.

Provenance and peer review Not commissioned; externally peer reviewed.

Open Access This is an Open Access article distributed in accordance with the Creative Commons Attribution Non Commercial (CC BY-NC 4.0) license, which permits others to distribute, remix, adapt, build upon this work non-commercially, and license their derivative works on different terms, provided the original work is properly cited and the use is non-commercial. See: http://creativecommons.org/ licenses/by-nc/4.0/

(C) Article author(s) (or their employer(s) unless otherwise stated in the text of the article) 2017. All rights reserved. No commercial use is permitted unless otherwise expressly granted.

\section{REFERENCES}

1. The World Health Organization. Active ageing: a policy framework. Madrid, Spain: WHO, 2002.

2. United Nations Department of Economic and Social Affairs Population Division. World population aging. New York: DESA, 2013.

3. Ibrahim R, Abolfathi Momtaz Y, Hamid TA. Social isolation in older Malaysians: prevalence and risk factors. Psychogeriatrics 2013;13:71-9.

4. Grenade L, Boldy D. Social isolation and loneliness among older people: issues and future challenges in community and residential settings. Aust Health Rev 2008;32:468-78.

5. Victor CR, Scambler SJ, Bowling ANN, et al. The prevalence of, and risk factors for, loneliness in later life: a survey of older people in great Britain. Ageing Soc 2005;25:357-75.

6. British Colombia Ministry of Health. Social isolation among seniors: an emerging issue, 2004.

7. Nicholson NR. A review of social isolation: an important but underassessed condition in older adults. J Prim Prev 2012;33:137-52.

8. Tomaka J, Thompson S, Palacios R. The relation of social isolation, loneliness, and social support to disease outcomes among the elderly. J Aging Health 2006;18:359-84.

9. Cacioppo JT, Hawkley LC, Norman GJ, et al. Social isolation. Ann N Y Acad Sci 2011;1231:17-22.

10. Cohen S, Doyle WJ, Skoner DP, et al. Social ties and susceptibility to the common cold. JAMA 1997;277:1940-4.

11. Cornwell EY, Waite LJ, Disconnectedness S. Perceived isolation, and Health among Older adults.. Journal of Health and Social Behavior 2009;50:31-48.

12. Hastings SN, George LK, Fillenbaum GG, et al. Does lack of social support lead to more ED visits for older adults? Am J Emerg Med 2008;26:454-61.

13. Landeiro F, Leal J, Gray AM. The impact of social isolation on delayed hospital discharges of older hip fracture patients and associated costs. Osteoporos Int 2016;27:737-45.

14. Mendieta M, Martin MAG. Effects of the social support groups on Ioneliness, social support and quality of life in the elderly. Revista de Psicologia Social Aplicada 2003;13:55-72.

15. Stewart M, Craig D, MacPherson K, et al. Promoting positive affect and diminishing loneliness of widowed seniors through a support intervention. Public Health Nurs 2001;18:54-63.
16. Dammeyer MM. Does social isolation among facility-dwelling elderly decrease using a reminiscence group intervention? Dissertation Abstracts International: section B: the sciences and engineering. 65, 2004. (5-B):2618.

17. Liu SJ, Lin CJ, Chen YM, et al. The effects of reminiscence group therapy on self-esteem, depression, loneliness and life satisfaction of elderly people living alone. Mid-Taiwan Journal of Medicine. 2007;12:133-42.

18. Tsai HH, Tsai YF, Wang HH, et al. Videoconference program enhances social support, loneliness, and depressive status of elderly nursing home residents. Aging Ment Health 2010;14:947-54.

19. Fokkema T, Knipscheer K. Escape loneliness by going digital: a quantitative and qualitative evaluation of a dutch experiment in using ECT to overcome loneliness among older adults. Aging Ment Health 2007;11:496-504.

20. Banks MR, Willoughby LM, Banks WA. Animal-assisted therapy and loneliness in nursing homes: use of robotic versus living dogs. J Am Med Dir Assoc 2008;9:173-7.

21. Jessen J, Cardiello F, Baun MM. Avian companionship in alleviation of depression, loneliness, and low morale of older adults in skilled rehabilitation units. Psychol Rep 1996;78:339-48.

22. Maclntyre I, Corradetti P, Roberts J, et al. Pilot study of a visitor volunteer programme for community elderly people receiving home health care. Health Soc Care Community 1999;7:2254p-232.

23. Stevens N, van Tilburg T. Stimulating friendship in later life: a strategy for reducing loneliness among older women.. Educational Gerontology 2000;26:15-35.

24. Alaviani M, Khosravan S, Alami A, et al. The effect of a Multi-Strategy program on developing Social Behaviors based on Pender's Health Promotion Model to Prevent Loneliness of Old Women Referred to Gonabad Urban Health Centers. Int J Community Based Nurs Midwifery 2015;3:132-40.

25. Robertson RJ, Banks OL. Indirect measurement of results in a project for improving socialization among the elderly. $J$ Gerontol 1970;25:265-7.

26. Wright L, Vance L, Sudduth $C$, et al. The impact of a HomeDelivered meal program on Nutritional Risk, Dietary Intake, Food Security, Loneliness, and Social Well-Being. J Nutr Gerontol Geriatr 2015;34:218-27.

27. Brennan PF, Moore SM, Smyth KA. The effects of a special computer network on caregivers of persons with Alzheimer's disease. Nurs Res 1995;44:166-72.

28. White $\mathrm{H}, \mathrm{McC}$ Connell $\mathrm{E}$, Clipp $\mathrm{E}$, et al. Surfing the net in later life: a review of the Literature and pilot study of computer use and Quality of Life. Journal of Applied Gerontology 1999;18:358-78.

29. Kahlbaugh PE, Sperandio AJ, Carlson AL, et al. Effects of playing Wi on Well-Being in the Elderly: physical activity, loneliness, and mood. Act Adapt Aging 2011;35:331-44.

30. Tse MM. Therapeutic effects of an indoor gardening programme for older people living in nursing homes. J Clin Nurs 2010;19.949-58.

31. Kremers IP, Steverink N, Albersnagel FA, et al. Improved selfmanagement ability and well-being in older women after a short group intervention. Aging Ment Health 2006;10:476-84.

32. Franck L, Molyneux N, Parkinson L. Systematic review of interventions addressing social isolation and depression in aged care clients. Qual Life Res 2016;25.

33. Dickens AP, Richards SH, Greaves CJ, et al. Interventions targeting social isolation in older people: a systematic review. BMC Public Health 2011;11:1-22.

34. Findlay RA. Interventions to reduce social isolation amongst older people: where is the evidence? Ageing Soc 2003;23:647-58.

35. Masi CM, Chen HY, Hawkley LC, et al. A meta-analysis of interventions to reduce loneliness. Pers Soc Psychol Rev 2011;15:219-66.

36. Hagan R, Manktelow R, Taylor BJ, et al. Reducing loneliness amongst older people: a systematic search and narrative review. Aging Ment Health 2014;18:683-93.

37. Choi M, Kong S, Jung D. Computer and internet interventions for loneliness and depression in older adults: a meta-analysis. Healtho Inform Res 2012;18:191-8.

38. Secretariat MA. Social isolation in community-dwelling seniors: an evidence-based analysis. Ont Health Technol Assess Ser 2008;8:1-49.

39. Sander R, Cattan M, White M, Bond J, Learmouth A. Preventing social isolation and loneliness among older people: a systematic review of health promotion interventions. Nurs Older People 2005;17:41-67.

40. Shamseer L, Moher D, Clarke M, et al. Preferred reporting items for systematic review and meta-analysis protocols (PRISMA-P) 2015: elaboration and explanation. BMJ 2015;349:g7647. 
41. Moher D, Liberati A, Tetzlaff J, et al. Preferred reporting items for systematic reviews and meta-analyses: the PRISMA statement. BMJ 2009;339:b2535.

42. Liberati A, Altman DG, Tetzlaff J, et al. The PRISMA statement for reporting systematic reviews and meta-analyses of studies that evaluate healthcare interventions: explanation and elaboration. BMJ 2009;339:b2700.

43. Welch V, Petticrew M, Tugwell P, et al. PRISMA-Equity 2012 extension: reporting guidelines for systematic reviews with a focus on health equity. PLoS Med 2012;9:e1001333.

44. Lubben JE. Assessing social networks among elderly populations. Fam Community Health 1988;11:42-52.

45. Bernard S. Loneliness and social isolation among older people in North Yorkshire, Working Paper (WP2565). Social Policy Research Unit, University of York 2013.

46. Powers JR, Goodger B, Byles JE. Assessment of the Abbreviated Duke Social support index in a cohort of Older Australian women. Australas J Ageing 2004;23:71-6.

47. de Jong-Gierveld J, Kamphuls F. The development of a Rasch-Type Ioneliness Scale. App/ Psychol Meas 1985;9:289-99.

48. Russell D, Peplau LA, Cutrona CE. The revised UCLA Loneliness Scale: concurrent and discriminant validity evidence. J Pers Soc Psychol 1980;39:472-80.

49. Masi CM, Chen H-Y, Hawkley LC, et al. A Meta-Analysis of interventions to reduce loneliness. Personality and Social Psychology Review 2011;15:219-66.
50. The Cochrane Collaboration. Data collection forms for intervention reviews: Rcts and non-RCTs . Version 3. 2014 (cited 2016 27th April) http://training.cochrane.org/resource/data-collection-formsintervention-reviews

51. McMaster University Faculty of Health Sciences. Quality assessment tool for quantitative studies In. Effective public health practice project. 2009 . http://www.ephpp.ca/tools.html. (cited 201627 April).

52. Armijo-Olivo S, Stiles CR, Hagen NA, et al. Assessment of study quality for systematic reviews: a comparison of the Cochrane Collaboration risk of Bias tool and the Effective Public Health Practice Project Quality Assessment Tool: methodological research. J Eval Clin Pract 2012;18:12-18.

53. Nicholson NR. Social isolation in older adults: an evolutionary concept analysis. J Adv Nurs 2009;65:1342-52.

54. Delisle M-A. What does Solitude mean to the Aged? Canadian Journal on Aging / La Revue canadienne du vieillissement 1988;7:358-71.

55. Peplau LA, Perlman D. Perspectives on loneliness. loneliness; $A$ sourcebook of current theory, research and therapy. UCLA: Los Angeles: Wiley-Interscience, 1982:1-18.

56. Victor $\mathrm{C}$, Scambler S, Bond J, et al. Being alone in later life: loneliness, social isolation and living alone. Rev Clin Gerontol 2000;10:407-17.

57. Lipsey MW, Wilson DB, Meta-Analysis P, et al. CA. Sage 2001.

58. The Cochrane Collaboration Cochrane HandbookforSystemaic Reviewsreviews of Interventionsinterventions 2011 . http://handbook cochrane.org/front_page.htm 